\title{
A new suite of twenty-two polymorphic microsatellite loci in the parasitic wasp, Habrobracon hebetor (Hymenoptera: Braconidae): Promising molecular tools for studying the population genetics of several beneficial braconid species
}

\author{
Madougou GARBA ${ }^{1}$, Anne LOISEAU ${ }^{2 A}$, LaURe BenOIT ${ }^{2 B}$ and Nathalie GAUTHIER ${ }^{2 C, *}$ \\ ${ }^{1}$ Direction Générale de la Protection des Végétaux, Ministère de l'Agriculture, BP323, Niamey, Niger; \\ e-mail: garba_madougou@yahoo.fr \\ 2 UMR (INRA ${ }^{\mathrm{A}} / \mathrm{IRD}^{\mathrm{C}} / \mathrm{Cirad}^{\mathrm{B}} /$ Montpellier SupAgro) Centre de Biologie pour la Gestion des Populations, 755 avenue du Campus \\ Agropolis, CS 30016, F-34988 Montferrier-sur-Lez, France; e-mails: loiseau@supagro.inra.fr, laure.benoit@cirad.fr, \\ nathalie.gauthier@ird.fr, nathalie.gauthier@supagro.inra.fr
}

Key words. Hymenoptera, Braconidae, Habrobracon hebetor, Bracon spp., biological control, parasitic wasp, microsatellite, population genetics, gene flow

\begin{abstract}
Combining a biotin-enrichment protocol and 454GS-FLX titanium pyrosequencing technology, we characterised 22 polymorphic microsatellite loci from the parasitic wasp, Habrobracon hebetor (Say) (Hymenoptera: Braconidae), a cosmopolitan species commonly used in biological control against a wide range of both major lepidopterous pests of stored products and field crops in different parts of the world. Three multiplex PCR sets were optimised and characterised across $46 \mathrm{H}$. hebetor specimens from two samples collected from millet fields in Niger. Two to 11 alleles were found per locus and observed heterozygosity ranged from 0.289 to 0.826 . Polymorphism was detected in both samples with a similar level of observed heterozygosity (0.482 vs. 0.502$)$ and number of alleles (4.1 vs. 3.6). Deviation from Hardy-Weinberg equilibrium was detected at the same five loci in both samples and five or seven more loci in each sample but was not associated with heterozygote deficiencies. Even though evidence for linkage disequilibrium was found between a few alleles, these new loci segregated independently. The variability of the 22 loci will enable estimates of genetic diversity and structure patterns, as well as gene flow between $\mathrm{H}$. hebetor populations at different spatial scales. Cross-species amplifications were successful among the six Bracon spp. tested and nine loci will be particularly appropriate for population genetic studies in $B$. brevicornis.
\end{abstract}

\section{INTRODUCTION}

Habrobracon hebetor (Say) (Hymenoptera: Braconidae) is a well-known gregarious ectoparasitoid of the larvae of a wide range of economically important moths that infest stored grains, nuts, and fruits as well as field crops in various parts of the world. This parasitoid is considered to be one of the most promising biological control (BC) agents for many stored-product pests, including the Indian meal moth Plodia interpunctella (Hübner), the Mediterranean flour moth, Ephestia kuehniella (Zeller), the rice moth Corcyra cephalonica (Stainton), the greater wax moth, Galleria mellonella L., the cotton bollworm Helicorverpa armigera (Hübner), and also the millet head miner, Heliocheilus albipunctella (de Joannis), thanks to its cosmopolitan distribution and ability to regulate populations of many destructive moth pests of stored-food commodities (Payne et al., 2011; Adarkwah et al., 2014; Ba et al., 2014; Ghimire \& Philipps, 2014). The parasitoid is sold commercially as a biological control agent, whilst because it can easily be mass reared, indigenous populations have also been reared and released in the field or in storage for effec- tive pest moth control in many parts of the world (Ghimire \& Philipps, 2010; Payne et al., 2011).

Many aspects of its general biology and ecology, and ways to use it in pest management, have already been thoroughly investigated (Antolin et al., 2003; Payne et al., 2011; Adarkwah et al., 2014; Ba et al., 2014; Ghimire \& Philipps, 2014). However, nothing is known about the population genetic structure or diversity of this widely used BC agent. Such knowledge would improve our understanding of the species and its populations at different spatial scales, provide useful pre- and post-information on the populations released in the framework of $\mathrm{BC}$ programmes, and facilitate design of more efficient control strategies. The knowledge gap underlines the need for discriminating molecular markers to accurately monitor $H$. hebetor in pest management programmes and for population genetics studies.

Microsatellite loci are powerful markers now commonly used for such purposes but, as far as we know, such markers have never been developed for $H$. hebetor or very closely related species (i.e. Bracon spp.) among the very large and diverse Braconidae fam-

\footnotetext{
* Corresponding author; e-mails: nathalie.gauthier@ird.fr, nathalie.gauthier@supagro.inra.fr
} 
ily. We thus decided in the present study to use a combination of biotin-enrichment and 454 pyrosequencing technologies, to isolate new microsatellite loci from $H$. hebetor. Of twenty-four microsatellite markers tested, 22 loci were found to be polymorphic and were subsequently tested and optimised in three multiplex reactions using 46 females from two natural populations sampled in Niger, West Africa. The cross reaction of these particular markers was also tested in six closely related braconids [Bracon brevicornis (Wesmael), B. nigricans (Szépligeti), B. cephi (Gahan), B. lissogaster (Muesebeck), and two Bracon $\mathrm{sp} p$.], some of which are also being used as $\mathrm{BC}$ agents in integrated pest management schemes to combat moth pests.

\section{MATERIAL AND METHODS}

\section{Sampling and DNA extraction}

Adult $H$. hebetor were collected in late summer 2014 from a pearl millet field in two localities in Niger located $80 \mathrm{~km}$ apart: Dantchandou $\left(13.40634^{\circ} \mathrm{N}, 2.71836^{\circ} \mathrm{E}\right)$ and Tondikoarey $\left(13.58236^{\circ} \mathrm{N}, 1.99969^{\circ} \mathrm{E}\right)$ (Table 1). All adults from the same locality were placed alive in a tube filled with $80 \%$ ethanol and sexed in the laboratory using a binocular microscope. In our study, a total of 46 females consisting of 24 females H. hebetor sampled from Dantchandou and 22 females from Tondikoarey, respectively, were then stored at $-20^{\circ} \mathrm{C}$ until molecular experiments.

Moreover, 61 females of six other related Bracon species were studied: $19 \mathrm{~B}$. brevicornis originated from a laboratory massrearing initiated with founders collected in 2006 near the city of Leipzig (Germany) and 24 B. nigricans from a laboratory massrearing initiated in 2015 with founders from Sicily (Italy), $12 B$. ceph $i$ and 4 B. lissogaster were collected in 2015 in the Montana (USA), and the two other Bracon spp. were collected in 2015 in the Ariège (South of France). All adults from the same locality and species were placed alive in a tube filled with $80 \%$ ethanol and sexed in the laboratory. Then, they were stored at $-20^{\circ} \mathrm{C}$ until molecular experiments.

Total genomic DNA of all females collected was individually extracted with the DNeasy Blood \& Tissue Kit (Qiagen, Courtaboeuf, France) according to the manufacturer's instructions.

\section{Microsatellite library, primer design and PCR amplification}

Total genomic DNA from 30 pooled individual $H$. hebetor was similarly extracted using the DNeasy Blood \& Tissue Kit (Qiagen) according to the manufacturer's instructions. Microsatellite loci were isolated at Genoscreen (Lille, France) using a biotinenrichment protocol adapted from Kijas et al. (1994). Production and pyrosequencing of enriched libraries for eight microsatellite motifs [i.e. $(\mathrm{TG})_{10},(\mathrm{TC})_{10},(\mathrm{AAC})_{8},(\mathrm{AAG})_{8},(\mathrm{ACG})_{8},(\mathrm{AGG})_{8}$, (ACAT $\left.)_{6},(\text { ACTC })_{6}\right]$ were carried out as described in Malausa et al. (2011). The resulting 39,189 sequences were subsequently sorted and selected using the open access QDD program (Meglécz et al., 2010). A total of 13,151 concatenated sequences longer than $100 \mathrm{bp}$ with motifs displaying at least six repeats and tandem-repetition-free-flanking regions were obtained, representing a huge source of potential markers. Nineteen to 22-bp long primers were designed using the web-based Primer3 algorithm (http://frodo. wi.mit.edu/primer3/, Rozen \& Skaletsky, 2000) implemented in QDD, and satisfied the following criteria (i) the target microsatellite had to have at least six repeats; (ii) the resulting PCR product had to be between 100 and 420 bp long; (iii) the flanking region had to contain at most a three-base mononucleotide stretch or two repeats of any di-hexa motif; (iv) the annealing temperature (Ta) was between $60^{\circ} \mathrm{C}$ and $63^{\circ} \mathrm{C}$, and the difference in $T a$ between the forward and the reverse primer was $\leq 1.5^{\circ} \mathrm{C}$; and (v) the self- complementarity of the primers and between the primers matched the quality criteria used as default parameters in Primer3.

Thirty-six primer pairs were selected. The resulting 36 potential new markers were BLASTed (http://blast.ncbi.nlm.nih.gov/ Blast.cgi) against previously published markers and none showed significant nucleotide identity with GenBank sequences. Consequently, they were optimised by monolocus PCR with DNA from eight females of diverse geographical origins. Genomic DNA was extracted from each individual $H$. hebetor using the DNeasy Tissue Kit (Qiagen). PCR amplifications were performed in a final volume of $10 \mu \mathrm{l}$ containing $5 \mu \mathrm{L}$ of the Qiagen multiplex PCR Master Mix (1X) (including Taq, $200 \mu \mathrm{M}$ of each dNTP and 1.5 $\mathrm{mM} \mathrm{MgCl}$ ), $2 \mu \mathrm{M}$ of primers, $1 \mu \mathrm{L}$ of genomic DNA, and $2.6 \mu \mathrm{L}$ of RNase-free water. All PCRs comprised the following steps: (i) initial denaturation at $95^{\circ} \mathrm{C}$ for $15 \mathrm{~min}$; (ii) 30 denaturation cycles at $94^{\circ} \mathrm{C}$ for $30 \mathrm{~s}$, annealing at $57^{\circ} \mathrm{C}$ for $90 \mathrm{~s}$, elongation at $72^{\circ} \mathrm{C}$ for $60 \mathrm{~s}$; and (iii) final elongation at $60^{\circ} \mathrm{C}$ for $30 \mathrm{~min}$. Twenty-four of the loci provided high quality amplifications with unambiguous allelic patterns and apparent polymorphism scored by agarose gel electrophoresis, and were retained for multiplex PCRs with fluorescently labelled primers. Using the Multiplex Manager v1.1 software (Holleley \& Geerts, 2009), they were arranged in three multiplex PCRs that minimised the formation of duplexes and maximised the range of amplification product sizes (Table 1).

\section{Genetic analyses}

To characterise the 24 loci, the 46 individuals collected in Niger were genotyped (Table 1). For analysis of microsatellite loci polymorphism, PCR amplifications of the extracted DNA were performed as described above. PCR products were separated on an ABI 3730 sequencer (Applied Biosystems, Montpellier, France). Allele sizes were scored against GeneScan TM 500 LIZ standard (Applied Biosystems) using GeneMapper ${ }^{\mathrm{TM}}$ v 4.0 software, and confirmed manually.

Levels of polymorphism [i.e. number of alleles observed $(\mathrm{Na})$, observed $\left(H_{O}\right)$ and unbiased expected $\left(H_{E}\right)$ heterozygosities, respectively], deviation from Hardy-Weinberg equilibrium (HWE) and linkage disequilibrium (LD) for each locus were estimated using GenePop 4.2 available on the web (Rousset, 2008; http:// genepop.curtin.edu.au/), both for each sample and globally over the 46 specimens. To correct for multiple comparisons, a sequential Bonferroni correction was applied for both LD and HWE tests. Where deviation from HWE was detected, departure to panmixia due to the presence of null alleles or/and scoring errors was estimated using the Micro-Checker program (van Oosterhout et al., 2004) and FreeNA package (Chapuis \& Estoup, 2007). To assess the level of genetic differentiation between both samples, pairwise $F_{S T}$ value over all loci was computed using FreeNA (with the ENA correction for null alleles) following 1000 bootstrapping replications over loci.

Cross-species amplification was tested using the three PCR multiplexes in the 61 females of six other related Bracon species, 19 B. brevicornis, 24 B. nigricans, 12 B. cephi, 4 B. lissogaster and 2 Bracon spp. PCR and genotyping conditions were as described above.

\section{RESULTS}

\section{Genetic diversity, Hardy-Weinberg and linkage testing}

At each locus, all $46 \mathrm{H}$. hebetor specimens provided a genotype (except for one specimen from Dantchandou at locus Heb3-08). Twenty-two loci out of the 24 tested were polymorphic, with the exception of Heb3-04 and Heb3-06). The number of alleles per locus ranged from two to 11 (average 4.667) and the observed heterozygosity over all specimens and loci from 0.289 to 0.826 (mean $H_{O}=0.491 \pm 0.218$ ). Polymorphism was detected in the 
Table 1. Characteristics of the microsatellite loci selected in Habrobracon hebetor. Allelic diversity, heterozygosity and null allele frequencies were estimated in 46 female specimens collected from two natural populations in Niger (Dantchandou $n=24$, Tondikoarey $n=22$ ).

\begin{tabular}{|c|c|c|c|c|c|c|c|c|c|}
\hline \multirow{2}{*}{$\begin{array}{l}\text { Locus } \\
\text { (GenBank } \\
\text { Accession no.) }\end{array}$} & \multirow{2}{*}{$\begin{array}{l}\text { Primer sequence }\left(5^{\prime}-3^{\prime}\right) \\
\text { (F: [dye]-forward; R: reverse) }\end{array}$} & \multicolumn{2}{|c|}{ Cloned allele } & \multirow{2}{*}{$\begin{array}{c}\frac{\text { Global }}{N a} \\
\text { ASR (bp) }\end{array}$} & \multicolumn{3}{|c|}{ Dantchandou $(n=24)$} & \multicolumn{2}{|c|}{ Tondikoarey $(n=22)$} \\
\hline & & $\begin{array}{l}\text { epeat } \\
\text { notif } \mathrm{si}\end{array}$ & $\begin{array}{l}\text { Allele } \\
\text { size (bp) }\end{array}$ & & & $a_{\left(P_{H W E} \text {-value }\right)} H_{0} / H_{E}$ & $f_{\text {null-allele }}$ & $\mathrm{Na} \underset{\left(P_{H W E}\right.}{H_{0} / H_{E}}$ & $f_{\text {null }}$ \\
\hline \multicolumn{10}{|c|}{ PCR multiplex set 1} \\
\hline $\begin{array}{l}\text { Heb2-02 } \\
\text { (KT966690) }\end{array}$ & $\begin{array}{l}\text { F: [PET]- CGTGCTAGCGGTGGAGTTAG } \\
\text { R: GCCTGTGCATCATCAAATGT }\end{array}$ & A) 13 & 118 & $\begin{array}{c}8 \\
108-130\end{array}$ & 7 & $\begin{array}{l}0.708 / 0.827 \\
(0.001)^{\star *}\end{array}$ & 48 & $5 \begin{array}{c}0.818 / 0.668 \\
(0.039)^{*}\end{array}$ & 0 \\
\hline $\begin{array}{l}\text { Heb3-01 } \\
\text { (KT966699) }\end{array}$ & $\begin{array}{l}\text { F: [VIC]- TGCACATTCCTTTACGTCCA } \\
\text { R: TGTTGACGATAGCGCTGGTA }\end{array}$ & $(\mathrm{ACC})_{9}$ & 127 & $\begin{array}{c}3 \\
119-128 \\
\end{array}$ & 3 & $\begin{array}{c}0.542 / 0.664 \\
(0.233)\end{array}$ & 0.057 & $3 \begin{array}{c}\mathbf{0 . 2 7 3 / 0 . 6 1 3} \\
(0.001)^{\star *}\end{array}$ & 0.197 \\
\hline $\begin{array}{l}\text { Heb2-13 } \\
\text { (KT966694) }\end{array}$ & $\begin{array}{l}\text { F: [VIC]- TGTGACGATGAGGAAACAGC } \\
\text { R: TCCGCTCACCATTTCCTTAC }\end{array}$ & C) $)_{13}$ & 191 & $\begin{array}{c}5 \\
185-193 \\
\end{array}$ & 5 & $\begin{array}{c}0.750 / 0.732 \\
(0.001)^{\star *}\end{array}$ & 49 & $2 \begin{array}{c}0.409 / 0.485 \\
(0 . .654)\end{array}$ & 0.045 \\
\hline $\begin{array}{l}\text { b3-04 } \\
\text { T9666701) }\end{array}$ & $\begin{array}{l}\text { F: [NED]- CTCCCTATCCTTGACATGCG } \\
\text { R: GGAGTTGACACCTGACGGAT }\end{array}$ & (CTA) & 182 & $\begin{array}{c}1 \\
180\end{array}$ & 1 & $-1-$ & 0 & $-1-$ & 0 \\
\hline $\begin{array}{l}\text { Heb2-17 } \\
\text { (KT966696) }\end{array}$ & $\begin{array}{l}\text { F: [6-FAM]- AATGGTGCGATGCTTAAAGG } \\
\text { R: CGGAGGTGAGCTTATGAAGG }\end{array}$ & $(\mathrm{CT})_{9}$ & 241 & $\begin{array}{c}11 \\
237-266 \\
\end{array}$ & 9 & $\begin{array}{c}\mathbf{0 . 6 6 7 / 0 . 7 6 7} \\
(1.000)\end{array}$ & 0.076 & $5 \begin{array}{c}0.727 / 0.707 \\
(0.001)^{\star *}\end{array}$ & 0 \\
\hline $\begin{array}{l}\text { Tb3-07 } \\
\text { T966703) }\end{array}$ & $\begin{array}{l}\text { F: [NED]- TGAGACGAC } \\
\text { R: ACACATCAACGCC }\end{array}$ & $(\mathrm{CTT})_{9}$ & 246 & $\begin{array}{c}4 \\
252-261 \\
\end{array}$ & 4 & $\begin{array}{c}0.667 / 0.645 \\
(0.001)^{\star \star}\end{array}$ & 5 & $2 \begin{array}{c}0.545 / 0.474 \\
(0.654)\end{array}$ & 0 \\
\hline $\begin{array}{l}3-09 \\
666705)\end{array}$ & $\begin{array}{l}\text { F: [VIC]- C/ } \\
\text { R: GGTTG }\end{array}$ & $(\mathrm{CAA})_{6}$ & 312 & $\begin{array}{c}4 \\
295-316 \\
\end{array}$ & 4 & $\begin{array}{c}0.250 / 0.364 \\
(0.027)^{*} \\
\end{array}$ & 0.066 & $3 \begin{array}{c}0.500 / 0.449 \\
(0.063)\end{array}$ & 0 \\
\hline T966704) & $\begin{array}{l}\text { F: [PET]- GGCAGCAGAATTAGCTGTTCA } \\
\text { R: CGGACAAATCTTGAAGGGAA }\end{array}$ & $(\mathrm{CGT})_{6}$ & 294 & $\begin{array}{c}3 \\
292-298 \\
\end{array}$ & 2 & $\begin{array}{l}0.521 / 0.510 \\
(1.00)\end{array}$ & 0 & $3 \begin{array}{c}\mathbf{0 . 0 4 6 / 0 . 5 2 1} \\
(0.001)^{\star \star}\end{array}$ & 0.307 \\
\hline $\begin{array}{l}\text { Heb2-25 } \\
\text { (KT966698) }\end{array}$ & $\begin{array}{l}\text { F: [6-FAM]- CACTGATTCTGAATGCTCATTAC } \\
\text { R: GCTTAGCCTGACGATATACATAG }\end{array}$ & $(f$ & 412 & $\begin{array}{c}3 \\
400-404\end{array}$ & 3 & $\begin{array}{l}0.333 / 0.595 \\
(0.07)\end{array}$ & 0 & $3 \begin{array}{c}0.727 / 0.638 \\
(0.419)\end{array}$ & 0 \\
\hline \multicolumn{10}{|c|}{ PCR multiplex set 2} \\
\hline 4 & $\begin{array}{l}\text { GCGCATATCAATATGTAGCATT } \\
\text { TGCATTATTCTCGC }\end{array}$ & $(A C)_{12}$ & 119 & $\begin{array}{c}4 \\
103-115 \\
\end{array}$ & 3 & $\begin{array}{c}0.417 / 0.529 \\
(0.325)\end{array}$ & 0.075 & $3 \begin{array}{c}0.364 / 0.613 \\
(0.001)^{\star *}\end{array}$ & 0.12 \\
\hline $\begin{array}{l}\text { b3-03 } \\
\text { T966700) }\end{array}$ & $\begin{array}{l}\text { F: [6-FAM]-ATTTGTACACCAGCC } \\
\text { R: AGCGTCAGCAGTAACAAGCA }\end{array}$ & C) $)_{7}$ & 17 & $\begin{array}{c}2 \\
175-178 \\
\end{array}$ & 2 & $\begin{array}{c}0.375 / 0.510 \\
(0.232)\end{array}$ & 0.083 & $\begin{array}{rr}2 & 0.727 / 0 \\
& (0.07 \\
\end{array}$ & 0 \\
\hline $\begin{array}{l}2-16 \\
966695) \\
\end{array}$ & $\begin{array}{l}\text { F: [VIC]- TGTGGGAACAGACAAAC } \\
\text { R: GGCTTTGTTTATGGCAAACC }\end{array}$ & $(A G)_{10}$ & 233 & $\begin{array}{c}5 \\
216-230 \\
\end{array}$ & 3 & $\begin{array}{c}0.500 / 0.457 \\
(0.331) \\
\end{array}$ & 0 & $4 \begin{array}{c}\mathbf{0 . 3 1 8 / 0 . 6 1 2} \\
(0.001)^{* *}\end{array}$ & 0.18 \\
\hline $\begin{array}{l}2-21 \\
966697)\end{array}$ & $\begin{array}{l}\text { F: [6-FAM]- } \\
\text { R: CTGCAT }\end{array}$ & $(\mathrm{CT})_{8}$ & 292 & $\begin{array}{c}4 \\
284-294 \\
\end{array}$ & 3 & $\begin{array}{c}0.417 / 0.465 \\
(0.033)^{*}\end{array}$ & 0.062 & $3 \begin{array}{c}0.318 / 0.487 \\
(0.104)\end{array}$ & 0.110 \\
\hline $\begin{array}{l}\mathrm{b} 4-02 \\
\text { r966706) }\end{array}$ & $\begin{array}{l}\text { F: [NED]- C } \\
\text { R: CAACT] }\end{array}$ & 17 & , & $\begin{array}{c}5 \\
309-325 \\
\end{array}$ & 5 & $\begin{array}{c}0.667 / 0.769 \\
(0.560)\end{array}$ & 057 & $\begin{array}{r}0.591 / \\
(0.6\end{array}$ & 0 \\
\hline $\begin{array}{l}2-06 \\
966692)\end{array}$ & $\begin{array}{l}\text { F: [NED]- GTGGCCACATCCTGTAATGA } \\
\text { R: CCGCTTGAACGATTTAGGAA }\end{array}$ & $(\mathrm{CA})_{17}$ & 133 & $\begin{array}{c}8 \\
114-160\end{array}$ & 8 & $\begin{array}{c}0.666 / 0.831 \\
(0.000)^{* *}\end{array}$ & 0.075 & $5 \begin{array}{c}1.000 / 0.799 \\
(0.002)^{\star *}\end{array}$ & 0 \\
\hline 66 & $\begin{array}{l}\text { F: [PET]- TCGA } \\
\text { R: TGGAGTCC }\end{array}$ & $(A G G)_{6}$ & 243 & $\begin{array}{c}1 \\
246 \\
\end{array}$ & 1 & $-1-$ & 0 & $-1-$ & 0 \\
\hline $\begin{array}{l}\text { Heb2-09 } \\
\text { (KT966693) }\end{array}$ & $\begin{array}{l}\text { F: [PET]- G } \\
\text { R: ATATCA }\end{array}$ & & 173 & $\begin{array}{c}6 \\
68-188 \\
\end{array}$ & 5 & $\begin{array}{c}0.417 / 0.481 \\
(0.050)\end{array}$ & 081 & $6 \begin{array}{c}0.455 / 0.691 \\
(0.001)^{* *}\end{array}$ & 0.10 \\
\hline \multicolumn{10}{|c|}{ PCR multiplex set 3} \\
\hline $\begin{array}{l}\text { eb3-05 } \\
\text { (T966710) }\end{array}$ & $\begin{array}{l}\text { F: [6-FAM]- GAGCAACATACGTGCGTCATA } \\
\text { R: GGAGACATAGTGGAGGCCAA }\end{array}$ & )$_{6}$ & 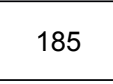 & $\begin{array}{c}3 \\
181-187 \\
\end{array}$ & 3 & $\begin{array}{c}0.375 / 0.549 \\
(0.170)\end{array}$ & 0.097 & $3 \begin{array}{c}0.636 / 0.580 \\
(0.078)\end{array}$ & 0 \\
\hline $\begin{array}{l}\text { eb4-01 } \\
\text { <T966709) }\end{array}$ & $\begin{array}{l}\text { F: [NED]- CTGTGGAGGGCTCGATTTAG } \\
\text { R: AGGAAGCGGGGATCCTATC }\end{array}$ & $(G G G A)_{6}$ & 612 & $\begin{array}{c}3 \\
117-129\end{array}$ & 3 & $\begin{array}{l}0.417 / 0.401 \\
(1.000)\end{array}$ & 0 & $-1-$ & 0 \\
\hline $\begin{array}{l}\text { Heb2-03 } \\
\text { (KT966707) }\end{array}$ & F: [6-FAM]- GTGGATGTTTCGTCACTGGA & ( & 118 & $\begin{array}{c}6 \\
103-113 \\
\end{array}$ & 3 & $\begin{array}{c}0.833 / 0.664 \\
(0.001)^{* *}\end{array}$ & 0 & $6 \begin{array}{c}0.773 / 0.776 \\
(0.001)^{\star *}\end{array}$ & 0 \\
\hline $\begin{array}{l}\text { Heb2-07 } \\
\text { (KT966708) }\end{array}$ & $\begin{array}{l}\text { F: [VIC]- CGCAGCTTCTGTGGAGTAAAC } \\
\text { R: TGTAAATGTCGCTACGTGCC }\end{array}$ & 11 & 140 & $\begin{array}{c}7 \\
137-149 \\
\end{array}$ & 6 & $\begin{array}{l}0.583 / 0.508 \\
(1.000)\end{array}$ & 0 & $5 \begin{array}{c}0.773 / 0.757 \\
(0.001)^{\star \star}\end{array}$ & 0 \\
\hline $\begin{array}{l}\text { Heb2-11 } \\
\text { (KT966712) }\end{array}$ & $\begin{array}{l}\text { F: [NED]- CAGCTGTAATATCAGCGGCA } \\
\text { R: CGGCAGATTGTAATGCGAGT }\end{array}$ & )$_{11}$ & 30 & $\begin{array}{c}5 \\
183-193\end{array}$ & 5 & $\begin{array}{c}\mathbf{0 . 5 8 3 / 0 . 7 8 4} \\
(0.000)^{\star *}\end{array}$ & 3 & $4 \begin{array}{c}0.773 / 0.684 \\
(0.351)\end{array}$ & 0 \\
\hline $\begin{array}{l}\text { Heb2-14 } \\
\text { (KT966711) }\end{array}$ & $\begin{array}{l}\text { F: [VIC]- TCCCTTCACCATGATCCATT } \\
\text { R: TGCATGAGCACTTGTTCAGA }\end{array}$ & $1 / 9$ & 202 & $\begin{array}{c}5 \\
193-209\end{array}$ & 4 & $\begin{array}{l}0.250 / 0.461 \\
(0.004)^{\star *}\end{array}$ & 0.132 & $4 \begin{array}{c}0.773 / 0.760 \\
(0.001)^{\star \star}\end{array}$ & 0 \\
\hline $\begin{array}{l}\text { T92-15 } \\
\text { T966714) }\end{array}$ & $\begin{array}{l}\text { F: [PET]- CATCGATCTTCATAAGTTTCC } \\
\text { R: CCCTGCATTGACTCAGGTC }\end{array}$ & $(A C)_{10}$ & 209 & $\begin{array}{c}6 \\
199-213\end{array}$ & 5 & $\begin{array}{c}0.625 / 0.655 \\
(0.005)^{*}\end{array}$ & 0 & $5 \begin{array}{c}0.500 / 0.661 \\
(0.001)^{\star *}\end{array}$ & 0 \\
\hline
\end{tabular}

Loci with significant deviations from Hardy-Weinberg expectations $\left(P_{H W E}<0.05^{*}, P_{H W E}<0.01^{* \star}\right)$ after Bonferroni correction. In bold significant heterozygote deficiency $(P<0.05)$. In bold $f_{\text {null-allele }}$ significant estimated null allele frequency per locus $\left(f_{\text {null-allele }}>0.15\right) . n-$ number of specimens studied; $\mathrm{Na}$ - number of alleles; ASR - allele size range; $H_{O}$ and $H_{E}$ - observed and expected heterozygosities, respectively; -l- irrelevant. 
Table 2. Successful cross-species amplifications of the microsatellite loci developed from Habrobracon hebetor in Bracon brevicornis, $B$. nigricans, B. cephi, B. lissogaster and two Bracon spp.

\begin{tabular}{|c|c|c|c|c|c|c|c|c|c|c|c|c|c|c|c|c|c|c|}
\hline \multirow[t]{2}{*}{ Locus } & \multicolumn{3}{|c|}{$\begin{array}{l}\text { Bracon brevicornis } \\
\quad(n=19)\end{array}$} & \multicolumn{3}{|c|}{$\begin{array}{l}\text { Bracon nigricans } \\
\quad(n=24)\end{array}$} & \multicolumn{3}{|c|}{$\begin{array}{l}\text { Bracon cephi } \\
\quad(n=12)\end{array}$} & \multicolumn{3}{|c|}{$\begin{array}{l}\text { Bracon lissogaster } \\
(n=4)\end{array}$} & \multicolumn{3}{|c|}{$\begin{array}{c}\text { Bracon sp1. } \\
(n=1)\end{array}$} & \multicolumn{3}{|c|}{$\begin{array}{l}\text { Bracon sp2. } \\
\quad(n=1)\end{array}$} \\
\hline & Nsa & $\mathrm{Na}$ & ASR (bp) & Nsa & $\mathrm{Na}$ & ASR (bp) & Nsa & $\mathrm{Na}$ & ASR (bp) & Nsa & $\mathrm{Na}$ & ASR (bp) & Nsa & $\mathrm{Na}$ & ASR (bp) & Nsa & $\mathrm{Na}$ & $\overline{A S R}(b p)$ \\
\hline Heb2-02 & 19 & 2 & $108-116$ & 24 & 1 & 108 & 0 & - & - & 0 & - & - & 0 & - & - & 0 & - & - \\
\hline Heb3-01 & 19 & 1 & 122 & 1 & 1 & 128 & 2 & 2 & $122-128$ & 0 & - & - & 0 & - & - & 0 & - & - \\
\hline Heb2-13 & 19 & 2 & $181-183$ & 0 & - & - & 0 & - & - & 0 & - & - & 0 & - & - & 0 & - & - \\
\hline Heb3-04 & 19 & 1 & 180 & 24 & 1 & 184 & 12 & 2 & 163-166 & 4 & 2 & $163-166$ & 0 & - & - & 1 & 2 & $163-169$ \\
\hline Heb2-17 & 19 & 1 & 253 & 24 & 2 & $227-229$ & 12 & 1 & 229 & 4 & 1 & 229 & 1 & 2 & $237-241$ & 1 & 1 & 227 \\
\hline Heb3-07 & 19 & 1 & 255 & 0 & - & - & 0 & - & - & 0 & - & - & 0 & - & - & 0 & - & - \\
\hline Heb3-09 & 19 & 1 & 313 & 0 & - & - & 0 & - & - & 0 & - & - & 0 & - & - & 0 & - & - \\
\hline Heb3-08 & 0 & - & - & 0 & - & - & 0 & - & - & 0 & - & - & 0 & - & - & 0 & - & - \\
\hline Heb2-25 & 18 & 1 & 401 & 0 & - & - & 0 & - & - & 0 & - & - & 0 & - & - & 0 & - & - \\
\hline Heb2-04 & 15 & 1 & 101 & 0 & - & - & 0 & - & - & 3 & 1 & 111 & 1 & 1 & 107 & 0 & - & - \\
\hline Heb3-03 & 19 & 1 & 175 & 6 & 3 & $175-181$ & 0 & - & - & 0 & - & - & 0 & - & - & 0 & - & - \\
\hline Heb2-16 & 19 & 1 & 226 & 24 & 1 & 222 & 8 & 1 & 230 & 0 & - & - & 0 & - & - & 1 & 1 & 228 \\
\hline Heb2-21 & 19 & 2 & 290-292 & 0 & - & - & 0 & - & - & 0 & - & - & 0 & - & - & 0 & - & - \\
\hline Heb4-02 & 19 & 1 & 317 & 0 & - & - & 0 & - & - & 0 & - & - & 0 & - & - & 0 & - & - \\
\hline Heb2-06 & 19 & 2 & $112-114$ & 24 & 1 & 110 & 0 & - & - & 0 & - & - & 0 & - & - & 0 & - & - \\
\hline Heb3-06 & 19 & 1 & 246 & 24 & 1 & 240 & 12 & 1 & 241 & 4 & 3 & $240-243$ & 1 & 1 & 243 & 1 & 1 & 250 \\
\hline Heb2-09 & 19 & 3 & $170-174$ & 0 & - & - & 0 & - & - & 0 & - & - & 0 & - & - & 0 & - & - \\
\hline Heb3-05 & 19 & 2 & 181-187 & 24 & 1 & 190 & 3 & 2 & 181-184 & 2 & 2 & $181-184$ & 1 & 2 & $181-190$ & 1 & 1 & 181 \\
\hline Heb4-01 & 19 & 2 & $125-129$ & 24 & 1 & 146 & 4 & 1 & 125 & 1 & 1 & 125 & 1 & 1 & 125 & 1 & 1 & 125 \\
\hline Heb2-03 & 19 & 1 & 105 & 24 & 1 & 110 & 7 & 4 & $105-113$ & 4 & 4 & $111-125$ & 0 & - & - & 1 & 1 & 105 \\
\hline Heb2-07 & 19 & 2 & $132-136$ & 24 & 1 & 121 & 3 & 3 & $132-138$ & 3 & 3 & $117-125$ & 0 & - & - & 0 & - & - \\
\hline Heb2-11 & 19 & 1 & 189 & 1 & 2 & 183-191 & 8 & 4 & 183-191 & 4 & 4 & 183-191 & 1 & 1 & 189 & 1 & 1 & 189 \\
\hline Heb2-14 & 19 & 2 & 197-199 & 22 & 1 & 190 & 5 & 4 & 194-200 & 2 & 2 & 198-200 & 0 & - & - & 1 & 1 & 200 \\
\hline Heb2-15 & 19 & 1 & 203 & 0 & - & - & 0 & - & - & 0 & - & - & 0 & - & - & 0 & - & - \\
\hline $\begin{array}{l}\text { Nstotal } \\
(\text { Npol) }\end{array}$ & & 2 & $\begin{array}{l}3 \\
9) \\
\end{array}$ & & $\begin{array}{l}1 \\
(3\end{array}$ & 14 & & 1 & $\begin{array}{l}11 \\
7) \\
\end{array}$ & & $\begin{array}{l}1 \\
(7 \\
\end{array}$ & $\begin{array}{l}0 \\
7) \\
\end{array}$ & & $\begin{array}{r}6 \\
(2 \\
\end{array}$ & & & $\begin{array}{r}9 \\
(1 \\
\end{array}$ & \\
\hline
\end{tabular}

$n$ - number of individuals tested; Nsa - number of successful and unambiguous amplifications/locus/species; Na - number of alleles; ASR - allele size range; Nstotal - number of successful loci amplified/species; Npol - number of polymorphic loci; “-” - irrelevant.

two samples, with a similar level of observed heterozygosity $\left(H_{O \text {-Dantchandou }}=0.482 \pm 0.215, H_{O \text {-Tondikoarey }}=0.502 \pm 0.290\right)$ and mean number of alleles $\left(\mathrm{Na}_{\text {Dantchandou }}=4.1, \mathrm{Na}_{\text {Tondikoarey }}=3.6\right)$ in both samples (Table 1). Only one fixed allele was observed at locus Heb4-1 in the Tondikoarey sample (Table 1). After Bonferroni correction, significant deviations from HWE were globally detected at five loci (Heb2-02, Heb2-06, Heb2-03, Heb214, Heb2-15) in both samples, the Tondikoarey sample exhibiting deviations at seven more loci and Dantchandou at five other loci (Table 1). From estimations made using the Micro-Checker and FreeNA softwares, there was no evidence of scoring errors and significant frequencies $(f>0.15)$ of null alleles, except for three loci in the Tondikoarey sample and one in the Dantchandou sample. After Bonferroni correction, evidence for LD was detected between a few alleles but they were not the same when considering Dantchandou and Tondikoarey samples separately (data not shown). The sampling itself rather than physical linkage between the loci may explain this result.

\section{Genetic differentiation}

The level of genetic differentiation between the samples from Dantchandou and Tondikoarey $\left(F_{S T(\mathrm{ENA})}=0.0981\right)$ was very low and statistically non-significant. In agreement with this, genetic assignment tests significantly assigned all genotyped individuals to the same genetic population (results not shown).

\section{Cross-species PCR amplification}

Cross-species amplifications were mostly successful. Twentythree loci out of the 24 selected loci from $H$. hebetor amplified in all B. brevicornis specimens, versus 11 in 22 out of 24 of the
B. nigricans specimens, 7 and 6 in at least $50 \%$ of the $B$. lissogaster and B. cephi specimens, respectively, and 9 and 6 in the two other Bracon spp. tested (Table 2). Interestingly, some of the loci amplified in $H$. hebetor displayed significant polymorphism in the related braconids tested, particularly in B. brevicornis with nine polymorphic loci, and $B$. cephi and $B$. lissogaster with seven polymorphic loci. Some alleles at some loci could also be species-diagnostic as they were fixed and private. For example, locus Heb2-16 had a fixed $226 \mathrm{bp}$ allele for B. brevicornis vs. alleles of $222 \mathrm{bp}$ for $B$. nigricans, whereas it failed to amplify using the DNA from B. lissogaster and Bracon sp.1 and provided other allele size ranges for H. hebetor and Bracon sp.2 (Table 2).

\section{DISCUSSION}

In our study, 22 polymorphic microsatellite markers were developed for the first time in the beneficial parasitic wasp, $H$. hebetor and - as far as we know - for closely related Braconids also.

Even though five loci of the 22 characterized displayed departures to HWE, no locus displayed significant heterozygote deficiencies nor significant LD. Both the mating behaviour and life history of $H$. hebetor promote outbreeding (in Antolin et al., 2003). But the individuals genotyped in our study were from small samples and on an agricultural crop, and may have been subject to selection and/or a possible population bottleneck. Inherent population fluctuations may contribute to the deviations from HWE observed at some loci. As a result, these new loci are potentially valuable for investigating genetic diversity and structure patterns of $H$. hebetor populations from different moth hosts 
in different environments and at different spatial scales around the world.

The low level of genetic differentiation among samples separated by distance of $\geq 80 \mathrm{~km}$ suggests intense gene flow at a regional scale. We are currently using our suite of microsatellite loci to genotype: (1) a large number of population samples collected at various locations in Niger and bordering countries of Sub-Saharan Africa to determine the large scale spatial genetic structure of this parasitic wasp species, as well as (2) some laboratory strains that were earlier released as part of biological control programmes against moth pests of stored products in order to enable monitoring of their genetic structure and population dynamics within the agroecosystem. Preliminary results (data to be presented in full elsewhere) reveal that these new loci provide useful information on $H$. hebetor dispersion at the field scale as it seems to be possible to locally differentiate some laboratory strains and wild strains, which will, we hope, eventually allow us to design more efficient biocontrol strategies against the target moth pest species in question. Furthermore, some of the loci here tested appear useable in population genetics studies involving related species such as $B$. brevicornis, and to a lesser extent, $B$. cephi and $B$. lissogaster, and be useful in distinguishing some of them in mixed populations.

ACKNOWLEDGEMENTS. This study was funded by the West Africa Agricultural Productivity Program (WAAP/PPAAO) in Niger and the Institut de Recherche pour le Développement (IRD), France through a collaborative programme between "La Direction Générale de Protection des Végétaux", Niamey and the IRD. Data used in this work were partly produced in the technical facilities of the Centre Méditerranéen Environnement Biodiversité in Montpellier, France. We thank L. Zappalà (Università degli Studi di Catania, Italy), T.A. Rand (USDA-ARS, Sidney, USA), A. Thiel (University of Bremen, Germany) and G. Delvare (CIRAD, Montpellier, France) for providing us with live samples of $B$. nigricans, B. cephi and B. lissogaster, B. brevicornis and the two Bracon spp. specimens, respectively. Lastly, we thank H.D. Loxdale for his helpful editorial comments on the manuscript. The authors declare that they have no conflict of interest.

\section{REFERENCES}

Adarkwah C., Ulrichs C., Schaarschmidt S., Badi B.K., Addai I.K., Obeng-Ofori D. \& Schöller M. 2014: Potential of Hymenopteran larval and egg parasitoids to control stored-product beetle and moth infestation in jute bags. - Bull. Entomol. Res. 104: 534-542.

Antolin M.F., Ode P.J., Heimpel G.E., O’Hara R.B. \& Strand M.R. 2003: Population structure, mating system, and sex-determining allele diversity of the parasitoid wasp Habrobracon hebetor. - Heredity 91: 373-381.
Ba M.N., Baoua I.B., Kabore A., Amadou L., Oumarou N., Dabire-Binso C. \& Sanon A. 2014: Augmentative on-farm delivery methods for the parasitoid Habrobracon hebetor Say (Hymenoptera: Braconidae) to control the millet head miner Heliocheilus albipunctella (de Joannis) (Lepidoptera: Noctuidae) in Burkina Faso and Niger - BioControl 59: 689-696.

Chapuis M.P. \& Estoup A. 2007: Microsatellite null alleles and estimation of population differentiation - Mol. Biol. Evol. 24: 621-631.

Ghimire M.N. \& Philipps T.W. 2010: Mass rearing of Habrobracon hebetor Say (Hymenoptera: Braconidae) on larvae of the Indian meal moth, Plodia interpunctella (Lepidoptera: Pyralidae): effects of host density, parasitoid density, and rearing containers. - J. Stor. Prod. Res. 46: 214-220.

Ghimire M.N. \& Philipps T.W. 2014: Oviposition and reproductive performance of Habrobracon hebetor (Hymenoptera: Braconidae) on six different pyralid host species. - Ann. Entomol. Soc. Am. 107: 809-817.

Holleley C.E. \& GeERTs P. 2009: Multiplex Manager1.0: a crossplatform computer program that plans and optimizes multiplex PCR. - Biotechniques 46: 511-517.

KiJas J.M.H., Fowler J.C.S., Garbett C.A. \& Thomas M.R. 1994: Enrichment of microsatellites from the citrus genom using biotinylated oligonucleotide sequences bound to streptavidincoated magnetic particles. — Biotechniques 16: 657-660.

Malausa T., Gilles A., Meglécz E., Blanquart H., Duthoy S., Costedoat C., Dubut V., Pech N., Castagnone-Sereno P., DÉLYE C. ET AL. 2011: High-throughput microsatellite isolation through 454 GS-FLX Titanium pyrosequencing of enriched DNA libraries. - Mol. Ecol. Res. 11: 638-644.

Meglécz E., Costedoat C., Dubut V., Gilles A., Malausa T., PeCh N. \& Martin J.F. 2010: QDD: a user-friendly program to select microsatellite markers and design primers from large sequencing projects. - Bioinformatics 26: 403-404.

Payne W., Tapsoba H., Baoua I.B., Malick B.N., N'Diaye M. \& DABIRE-BInso C. 2011: On-farm biological control of the pearl millet head miner: realization of 35 years of unsteady progress in Mali, Burkina Faso and Niger. - Int. J. Agr. Sustainability 9: $186-193$

Rozen S. \& Skaletsky H.J. 2000: Primer3 on the web for general users and biologist programmers. In Krawetz S. \& Misener S. (eds): Bioinformatics Methods and Protocols: Methods in Molecular Biology. Humana Press, Totowa, NJ, pp. 365-368.

Rousset F. 2008: Genepop'007: a complete re-implementation of the Genepop software for Windows and Linux. - Mol. Ecol. Res. 8: 103-106.

Van Oosterhout C., Hutchinson W.F., Wills D.P.M. \& Shipley P. 2004: Microchecker: software for identifying and correcting genotyping errors in microsatellite data. - Mol. Ecol. Notes 4: $535-538$.

Received December 16, 2015; revised and accepted January 13, 2016 Published online March 7, 2016 